\section{PREOPERATIVE AND INTRAOPERATIVE ULTRASONOGRAPHIC EXAMINATION AS AN AID IN LUNG CANCER OPERATIONS}

To assess the extent of tumor invasion in lung cancer, my colleagues and I routinely use preoperative transesophageal ultrasonic endoscopy and intraoperative ultrasonography in addition to preoperative roentgenography, computed tomographic scanning, and other standard procedures. Both transesophageal ultrasonic endoscopy and intraoperative ultrasonography allow for a real-time assessment of the extent to which the lung cancer has invaded adjacent organs and are useful in determining the operability and safety margin of the involved organ or organs. We found intraoperative ultrasonography to be more accurate than transesophageal ultrasonic endoscopy, because intraoperative ultrasonography can be done at any time during the operation, as needed, and the probe can be directly applied to the desired location from a variety of angles. In contrast, when transesophageal ultrasonic endoscopy is used, the presence of air in the lung tissue can interfere with an accurate evaluation of some aspects of the tumor. Our results indicate that the sensitivity of transesophageal ultrasonic endoscopy and intraoperative ultrasonography is $68.4 \%$ and $100 \%$, respectively, and the specificity is $81.3 \%$ for transesophageal ultrasonic endoscopy and 95.5\% for intraoperative ultrasonography. (J THORAC CARDIOVASC SURG 1995;110:606-12)

Toshiki Tatsumura, MD, PhD, Toyama, Japan
$R_{h}$ ecent advances in perioperative management have introduced more aggressive approaches to the combined surgical treatment of cases of lung cancer in which the tumor has invaded adjacent related organs.

Combined resection of the involved chest wall in lung cancer has provided a favorable 5 -year survival of $20 \%$ to $35 \% .^{1-4}$ Smith $^{5}$ reported a good surgical result in patients with involvement of the left atrium, and my colleagues and $\mathrm{I}^{4}$ have observed a 5-year survival of $47.8 \%$ in patients with pericardial involvement. We also reported on a patient with combined resection of the left atrium and the presence of positive mediastinal nodal metastases; the patient is still alive and free of disease 7 years and 4

From the Department of Emergency, Toyama Medical and Pharmaceutical University, Toyama, Japan.

Presented in part at the Sixth World Conference of Lung Cancer, Melbourne, Australia, November 1991.

Received for publication Sept. 2, 1994.

Accepted for publication Dec. 20, 1994.

Address for reprints: Toshiki Tatsumura, MD, PhD, FCCP, FICA, Emergency Department, Toyama Medical and Pharmaceutical University, 2630 Sugitani, Toyama 930-01, Japan.

Copyright (C) 1995 by Mosby-Year Book, Inc.

$0022-5223 / 95 \$ 5.00+0 \quad \mathbf{1 2 / 1 / 6 3 1 7 7}$ months after operation. ${ }^{4}$ However, in cases involving the great blood vessels, the results of surgical treatment have been poor. ${ }^{4,6}$ In view of these results, it is essential to carefully select patients before applying such aggressive combined resection procedures. The use of ultrasonographic techniques, both preoperatively with transesophageal ultrasonic endoscopy (EUS) and intraoperatively with intraoperative ultrasonography (IUS), makes it possible to accurately assess the extent to which the primary lung cancer has invaded the adjacent organs. This significantly assists in the determination of preoperative staging, the operability of the cancer, and the range of resection. In the present study, I assessed the diagnostic sensitivity, specificity, and accuracy of these two techniques as applied to lung cancer operations.

\section{Patients and methods}

Since early 1980, both EUS and IUS have been integrated into the routine preoperative examination protocol for all patients with lung cancer, and IUS has been used intraoperatively where invasion of one or more adjacent organs was observed or suspected. I used a ToshibaMachida (Tokyo, Japan) EPB-503BL ultrasonic endoscope, which uses a linear scanning mode with a central frequency of $5 \mathrm{MHz}$. Images were displayed on a Toshiba SAL-50A monitor. An Aloka SSD-330 (Tokyo, Japan) 
Table I. Accuracy of EUS in assessing the existence of mediastinal invasion by a lung tumor

\begin{tabular}{ccc} 
& \multicolumn{2}{c}{ Pathologic diagnosis } \\
\cline { 2 - 3 } EUS & Invasion & No invasion \\
\hline Invasion & 13 & 3 \\
No invasion & 6 & 13 \\
Total & 19 & 16 \\
\hline
\end{tabular}

instrument was used for IUS, and the frequency used was either 5.0 or $7.5 \mathrm{MHz}$. The probes used for IUS included a linear type $\mathrm{T}$ and type I and a convex type. Ultrasonograms were recorded with video and Polaroid cameras.

As with a conventional endoscopic examination of the upper gastrointestinal tract, the patient is instructed to fast before EUS. The ultrasonic endoscope is inserted orally and rotated 360 degrees around the entire mediastinum to examine the condition of mediastinally located organs, including the lymph nodes. The diagnostic criteria for a positive finding of mediastinal involvement are as follows: (1) ultrasonographic findings show the tumor extending into a mediastinal organ or organs, (2) deformation or disappearance of the histologic structural layer of adjacent organs is observed, or (3) movement associated with normal respiratory or cardiac motion is not seen during the real-time observation. The presence of any of these findings suggests tumor invasion into mediastinal organs.

IUS is done by applying the probe directly to the tumor and related adjacent organs during the operation. Hence the probe or probes can be applied at any time and from any direction during the operative procedure to accurately assess the extent of the disease. The procedure can be done by directly pressing the probe firmly against the target organ or organs or through a water bag. The diagnostic criteria are the same as those for EUS.

In the present study, 71 patients with primary lung cancer were subjected to EUS before the operation to assess the extent of tumor invasion into the mediastinal organs. All of these patients eventually underwent an operation, and all of the resected specimens were carefully examined for histopathologic evidence of invasion of the related organ or organs by the primary lung tumor. IUS was used in 34 cases in which invasion of the primary lung cancer to the surrounding organs was observed or suspected. These included cases of centrally located lung cancer with involvement of mediastinal organs and peripheral lung cancer with chest wall involvement. In these cases, IUS was used as an aid in the determination of resectability of the tumor and the amount of tissue to be resected because the extent of the involvement of the related organ or organs was difficult to precisely assess by visual inspection or palpation alone. Informed consent was obtained from each patient before any of these procedures were done.

\section{Results}

Among the 71 cases studied, EUS was only able to detect primary lung tumors in 35 cases. This is
Table II. Accuracy of IUS in assessing the existence of tumor invasion of adjacent organs

\begin{tabular}{ccc} 
& \multicolumn{2}{c}{ Pathologic diagnosis } \\
\cline { 2 - 3 } Ins & Invion & No invasion \\
\hline Invasion & 12 & 1 \\
No invasion & 0 & 21 \\
Total & 12 & 22 \\
\hline
\end{tabular}

because EUS can only detect those tumors that are medially located in the vicinity of the esophagus: other locations are obscured by the air in the lung. Table I shows the results of the EUS examinations of these 35 cases. In 16 cases, the EUS examination led to a preoperative diagnosis of mediastinal organ invasion by the primary tumor. Of these 16 cases, 13 were confirmed to involve mediastinal organ invasion on pathologic examination of the resected tissue. However, in the remaining 3 cases, no tumor invasion of the mediastinal organs was found. In 19 cases, the EUS examination led to a preoperative diagnosis of negative mediastinal organ invasion by the primary tumor. In 13 of these cases, pathologic examination of the resected tissue confirmed this diagnosis. However, in 6 cases in which the original diagnosis found no mediastinal organ involvement, tumor invasion was eventually found during pathologic examination of the resected tissues. In this study, the sensitivity of EUS was $68.4 \%$, the specificity was $81.3 \%$, and the accuracy was $74.3 \%$. In addition, false-positive (18.8\%) and false-negative (31.6\%) findings were prevalent with this method (Table I).

In contrast, in 12 of the 13 cases in which IUS led to a positive diagnosis of tumor invasion of nearby organs, the diagnosis was confirmed on pathologic examination of the resected tissue (Table II). With IUS, there was only one false-positive diagnosis out of $13(7.7 \%)$ and there were no false-negative results. In this study, the sensitivity rate of this technique in assessing the extent of tumor invasion was $100 \%$; the specificity was $95.5 \%$ and the accuracy was $97.1 \%$. This suggests that IUS is much more reliable than EUS in the evaluation of primary lung tumor invasion of adjacent organs.

Several typical examples of the echographic features revealed by EUS and IUS are illustrated in Figs. 1 through 4 .

\section{Discussion}

In the combined resection of adjacent organs during lung cancer operation, an accurate preoper- 

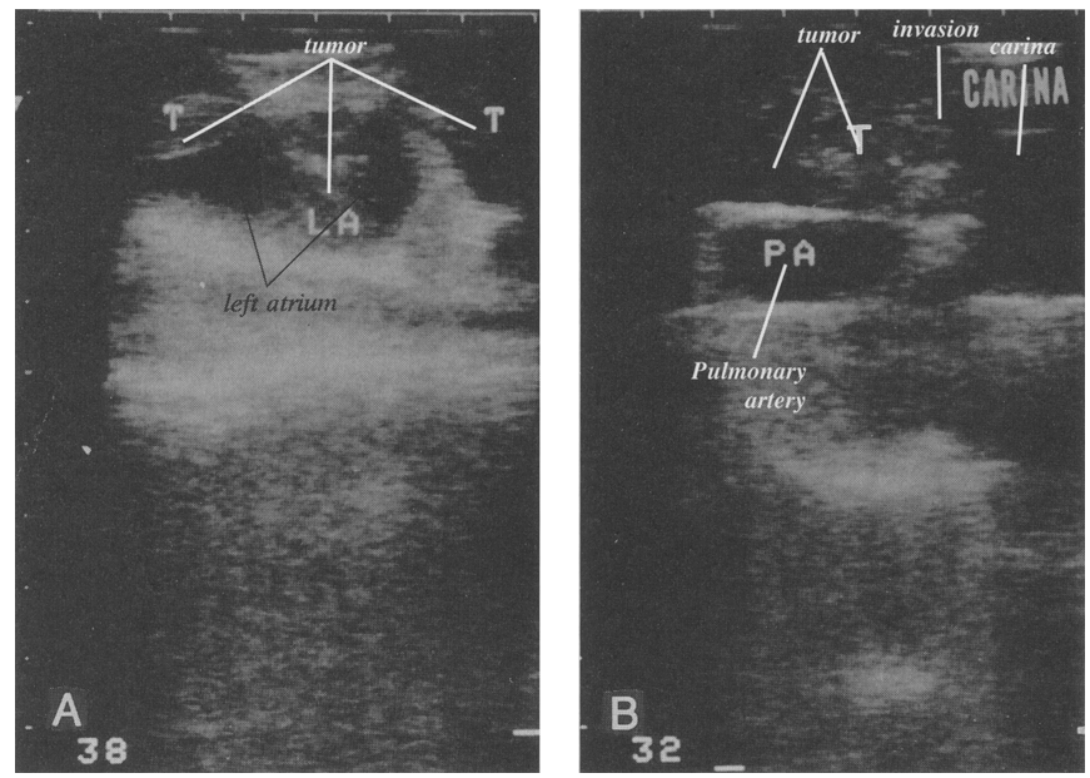

Fig. 1. A, Real-time preoperative examination by EUS clearly revealed that a large part of the left atrium $(L A)$ was invaded by a hilar-originating squamous cell carcinoma $(T)$. Intrusion of the tumor into the atrial cavity was noted in this ultrasonogram. Serial observations by EUS clearly indicated that, to extirpate the tumor, a large portion of the left atrium had to be resected, which would result in incompetence of the left atrium. This information prevented unnecessary operative intervention in this patient. B, Moreover, when the fiberscope was retracted orally, the primary tumor $(T)$ was also found to have invaded the carina, and the right main pulmonary artery $(P A)$ was elevated anteriorly.

ative assessment of the extent of tumor invasion into the adjacent organ or organs is important. The organs that may be involved include the pericardium, aorta, superior vena cava, main pulmonary vessels, left atrium, chest wall, and diaphragm, among others. Nevertheless, routine conventional examination methods such as chest roentgenography, computed tomographic (CT) scanning, angiography, bronchography, magnetic resonance imaging (MRI), and other methods sometimes yield insufficient information for an accurate preoperative determination of the extent of the invasion of the tumor into mediastinal organs. Even during an operation, the exact extent of invasion of a tumor into adjacent organs can sometimes be difficult to determine by visual inspection or palpation alone. This can sometimes cause an overestimation or underestimation of the extent of the cancer, and too little or too much tissue may be removed. In our department we apply EUS to all patients with lung cancer before operation and routinely use IUS intraoperatively on those patients in whom tumor invasion into an adjacent organ or organs is observed or suspected. This allows us to accurately evaluate the extent to which the primary tumor or metastatically affected nodes have invaded the mediastinal organs or the chest wall and to determine the operability of the tumor and the optimal range of resection. Moreover, since early $1980,{ }^{4,7-9}$ we have used EUS in all patients with lung cancer, in addition to $C T$ scanning and MRI, to more accurately determine the mediastinal staging of the lymph nodes.

The present study demonstrates that both EUS and IUS are extremely valuable tools for the preoperative and intraoperative assessment of lung cancers. However, although EUS was superior in detecting tumor invasion to mediastinal organs such as the main pulmonary vessels, the aorta, the cardiac compartment (especially the left atrium), and those organs located in the subcarinal and aortopulmonary window area, it was unreliable in the assessment of those organs located away from these areas.

The use of EUS in the assessment of mediastinal involvement and lymph node metastasis associated with lung cancer is rarely noted in the literature. To my knowledge there have been only two such re- 


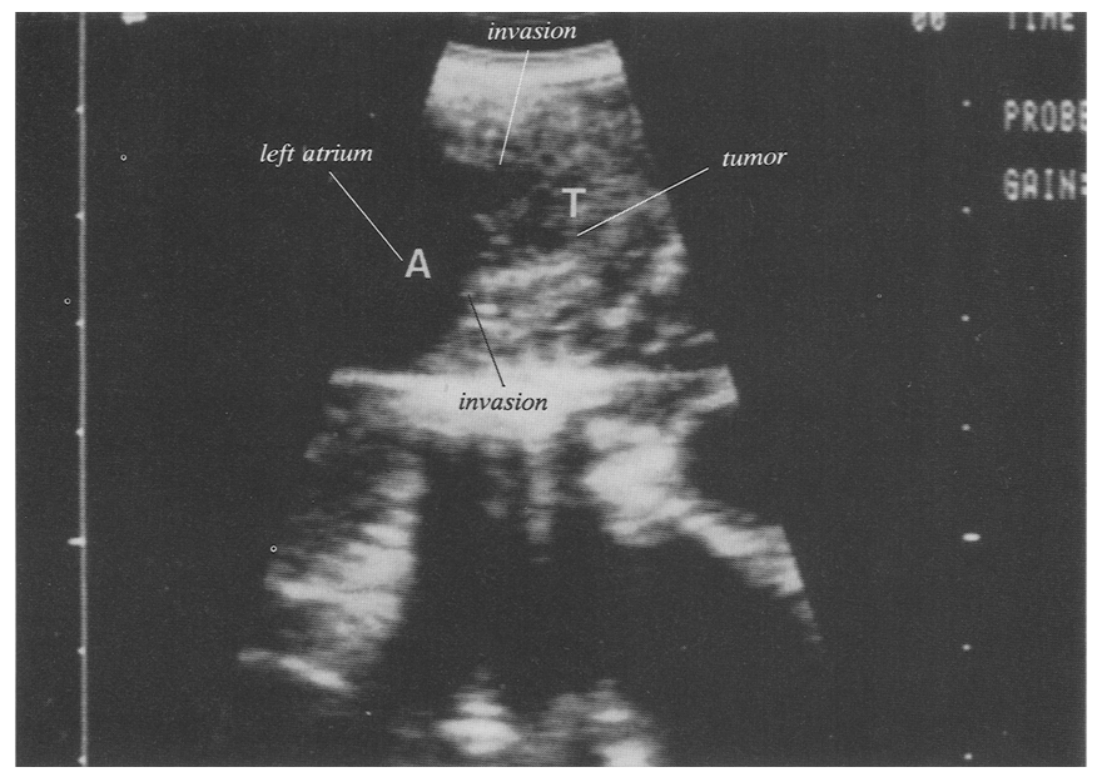

Fig. 2. Squamous cell carcinoma of the left hilum with invasion of the left atrium (IUS). Invasion of the left atrium $(A)$ by the tumor $(T)$ is clearly seen in this echogram. Note the irregular notches and indentation of the invaded atrial wall produced by the infiltration of the tumor. No movement of the atrial wall at the edge of the tumor invasion could be seen during real-time observation. The IUS observations in this patient confirmed that combined resection of the atrial wall was possible and the operation was done successfully with use of large vascular clamp.

ports, one of which concerns a case that involved aortic infiltration by lung cancer, ${ }^{10}$ whereas the other described a small number of cases in which EUS was used to evaluate lymph node metastasis. ${ }^{11}$

Intraoperative application of ultrasonography during lung cancer operation has been reported by Machi and associates. ${ }^{12}$ They assessed the extent of cardiovascular invasion by the tumor during lung cancer operation and reported an accuracy of 91.7\%. They compared this method with preoperative examination with CT scanning and angiography and found intraoperative use of ultrasonography to be far superior. However, no detailed information was provided in these later two studies. In the present study, I found that IUS had an accuracy of $97.1 \%$ and a specificity and sensitivity greater than $95 \%$. This is in good agreement with our previous results ${ }^{4,8}, 9$ and further confirms the usefulness of IUS in the perioperative analysis of lung tumors. Therefore I strongly recommend the routine use of this technique in the practice of lung cancer surgery, particularly in those patients in whom tumor invasion of the adjacent organ or organs or lymph node metastasis is observed or suspected.

In this study, the diagnostic sensitivity of EUS
(68.4\%) was found to be low, with a particularly high prevalence of false-negative results in cases in which the tumor had invaded the pericardium or mediastinal pleura. However, this is close to the sensitivity of more conventional examination methods such as CT scanning and MRI. Musset and colleagues ${ }^{13}$ used CT scanning and MRI to evaluate cases of T3 disease and found that the sensitivity of these two methods was only $53 \%$ and $60 \%$, respectively. Webb and colleagues ${ }^{14}$ evaluated the ability of CT scanning and MRI to distinguish T3 and T4 tumor locations from the size of the tumor and reported a sensitivity of $63 \%$ and a specificity of $84 \%$ for CT scanning and a sensitivity of $56 \%$ and a specificity of $80 \%$ for MRI. Similarly, Martini and associates ${ }^{15}$ used these methods to evaluate tumor invasion into the mediastinal organs and found a sensitivity of $55 \%$ for CT scanning and $64 \%$ for MRI. There was no consistently significant difference between the sensitivity of CT scanning and MRI in these studies. Nevertheless, Fig. 1 shows that EUS can be useful in some cases in the preoperative determination of the extent of the tumor and its operability. This can alleviate the need for many unnecessary exploratory surgical procedures in some cases. Nevertheless, 


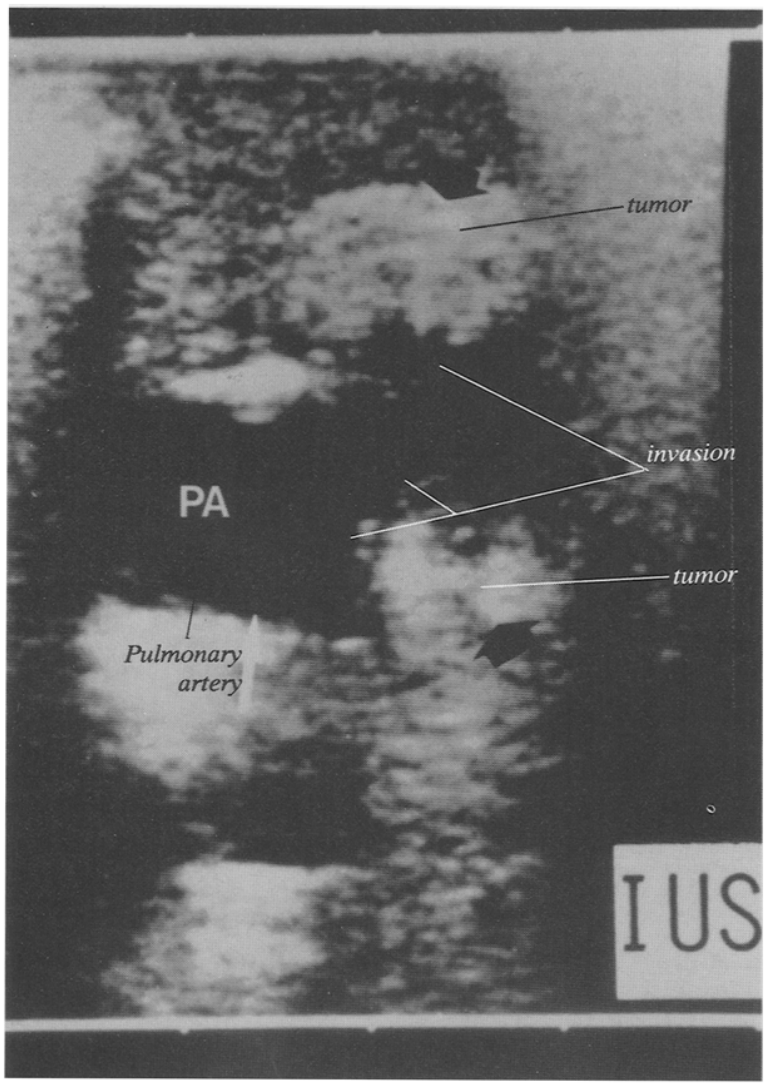

Fig. 3. Squamous cell carcinoma of the right hilum (IUS). The large tumor had spread over the right main pulmonary artery $(P A)$. It was difficult to outline the actual extent of the whole tumor into the involved pulmonary artery by palpation and observation alone because of size of the tumor. However, as shown here, the main pulmonary artery (white arrow) was found to be nearly intact and invasion of the tumor (black arrows) to peripheral parts of the pulmonary artery was clearly revealed as severe uneven notches or a "mothy" appearance of the regions that had been invaded by the tumor. In this case, IUS was able to provide the information necessary to determine the operability of the tumor and the appropriate extent of resection of the involved tissue. A pneumonectomy was done in this case.

EUS is useful for the determination of the extent of tumor invasion into tissue in the vicinity of the esophagus.

In cases in which the chest wall has been invaded by lung cancer, it is sometimes difficult to reliably assess the exact extent of the invasion of the tumor, especially when the disease is complicated by inflammatory adhesion between the tumor and the involved organ or organs. In such cases, overestimation or underestimation of the extent of the invasion

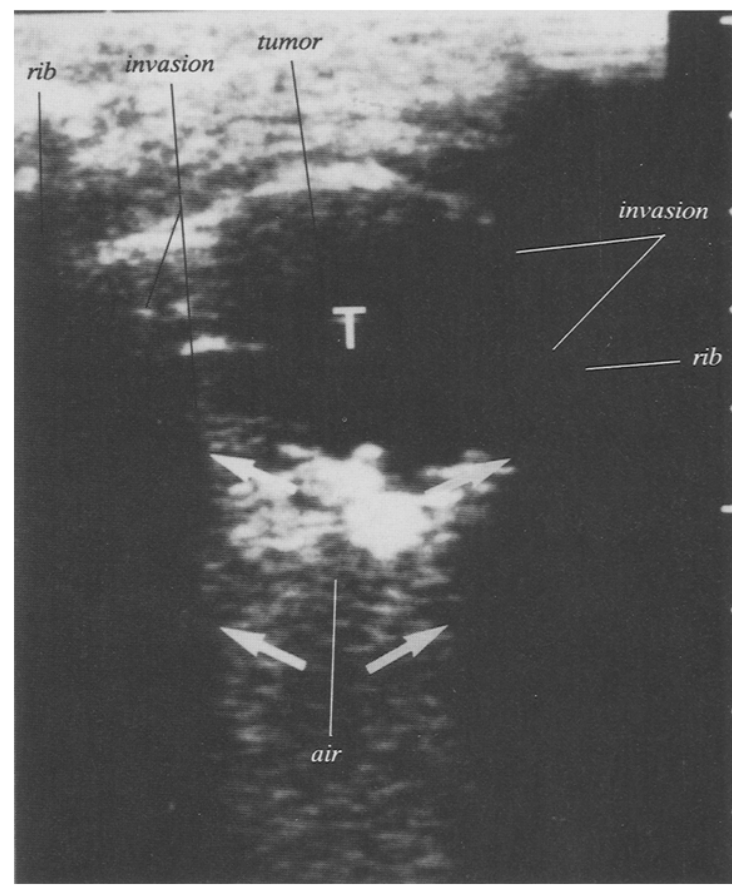

Fig. 4. Peripherally located squamous cell carcinoma with invasion to the ribs (IUS). This real-time echogram was done after the prethoracic tissues were opened. The probe was directly aimed at the involved bare chest wall. The ribs (arrows) were severely invaded by the tumor $(T)$, which extended into the marrow portion of the involved ribs and into the intercostal muscles. Moving the probe between the invaded and normal portions of the rib during the operation allowed assessment of safety margin of the rib.

may occur with roentgenographic or CT preoperative examinations or with intraoperative palpation. Moreover, correct assessment of the invasion to the periosteum, bony structures, or bone marrow portion of the ribs is difficult with use of the conventional methods described. In such cases transthoracic ultrasonography and IUS would be useful in distinguishing the extent of the invasion of the tumor and in determining the safety margin of the ribs to be resected (Fig. 4). One of the significant advantages of IUS is that it can be applied repeatedly throughout the operation and from any angle necessary to obtain the desired information about the tumor. An IUS examination is of similar benefit in the assessment of the degree to which mediastinally located organs have been invaded by the tumor.

Usually, tumors that have invaded the ventral and lateral portions of the left atrium can be easily 
defined after the pericardium is opened. However, when there is extensive tumor invasion involving the dorsal aspect of the atrium (as in Fig. 2), it is almost impossible to assess the entire extent of the tumor by palpation alone. The use of IUS allows the entire contour of the tumor within the atrium to be clearly outlined. In one such case (Fig. 2), the atrium was successfully resected in combination with the tumor by use of a large vascular clamp. Cardiopulmonary bypass was not used in this case, and the patient is still alive 7 years and 4 months after the operation, with no evidence of recurrence of the tumor or of distal metastasis.

In the case illustrated in Fig. 3, cancerous and inflammatory processes resulted in adhesion and invasion of the tumor to the surrounding tissues, and shortening of the main pulmonary artery was noted. These features and the large size of the tumor made it difficult for the surgeon to assess the precise extent of tumor invasion into the main pulmonary artery, its branches, and surrounding tissues. However, with the assistance of IUS, the surgeon was able to assess the extent of the tumor without performing the extensive dissections that would have been necessary to allow the tumor to be reached for palpation. This prevented unnecessary surgical intervention in the patient. However, in less advanced cases, with less pronounced tumor invasion, palpation or visualization will usually be sufficient to define tumor invasion.

Moreover, the diagnostic criteria for a positive finding of mediastinal involvement by use of an ultrasonogram (see Methods section) are applicable to all cases with tumor invasion, including those cases with involvement of the great vessels such as the aorta or superior vena cava. Diagnosis of intrusion of the tumor into these vessels, and the disappearance or deformation of the vascular wall, is greatly facilitated by the use of IUS. Without IUS, the adhesion to, or invasion of, the adventitia by the tumor may be underestimated or overestimated.

Suzuki, Saitoh, and Kitamura ${ }^{16}$ compared the diagnostic efficacy of ultrasonography and CT scanning in the evaluation of chest wall invasion by lung cancer. They reported that ultrasonography was far superior to CT scanning, with a high sensitivity of $100 \%$, a specificity of $98 \%$, and an accuracy of $98 \%$. Moreover, Glazer and associates, ${ }^{17}$ in their review of the efficacy of CT scanning in the assessment of chest wall invasion, reported a sensitivity of $87 \%$ and a disappointing specificity of only $59 \%$. Pennes and colleagues ${ }^{18}$ found CT scanning to be even less effective, with a sensitivity of only $38 \%$ a specificity of $40 \%$, and an accuracy of $39 \%$.

In lung cancer complicated by invasion to adjacent organs, an improved prognosis can be achieved by a combined resection of the involved organ or organs together with the lung cancer and en bloc lymph node dissection in selected cases. Ultrasonography, which is a noninvasive technique when done by transthoracic methods or IUS, should be actively adopted as a routine practice in the treatment of patients with lung cancer with invasion of the tumor into an adjacent organ or organs. EUS is also well tolerated by patients if it is done by a skilled physician. Both of these techniques, combined with conventional methods, can significantly improve the ability of the surgeon to make accurate decisions concerning patient treatment, the optimal operative method, and the safety margin of the resection.

I thank Dr. M. Kasashima and Dr. S. Koyama for their technical assistance and Miss K. Fujii for her assistance in the preparation of this manuscript.

\section{REFERENCES}

1. Geha AS, Bernatz PE, Woolner LB. Bronchogenic carcinoma involving the thoracic wall: surgical treatment and prognostic significance. J THORAC CARIIOVASC SURG 1967;54:394-402.

2. Mountain CF. Assessment of the role of surgery for control of lung cancer. Ann Thorac Surg 1977;24:365-73.

3. Allen MS, Mathisen DJ, Grillo HC, et al. Bronchogenic carcinoma with chest wall invasion. Ann Thorac Surg 1991;51:948-51.

4. Tatsumura T, Kasashima M, Koyama S, et al. Problems in combined resection of adjacent organ in lung cancer: significance of preoperative and intraoperative ultrasonic examination [in Japanese]. Jpn J Thorac Surg 1992;45:1155-62.

5. Smith RA. The results of raising the resectability rate in operations for lung carcinoma. J THORAC CARDIOVASC SuRg 1964;48:418-26.

6. Bailey CP, Schechter DC, Folk FS. Extending operability in lung cancer involving the heart and great vessels. Ann Thorac Surg 1971;11:140-50.

7. Kasashima M, Yamamoto K, Tatsumura T, et al. The diagnosis of mediastinal invasion of lung cancer with transesophageal ultrasonic endoscopy [in Japanese]. Image Technol Information Display 1986;18:989-94.

8. Tatsumura T, Kasashima M. Significance of pre and intraoperative ultrasonographic diagnosis of mediastinal nodal metastasis in lung cancer. In: Naruke $T$, ed. Principles of lung cancer surgery [in Japanese]. Vol. 1. Tokyo: Society for Study on Principle of Lung Cancer Surgery, 1989:218-33.

9. Tatsumura T, Kasashima M. Metastasis of lung can- 
cer to mediastinal lymph nodes: preoperative and intraoperative diagnoses by ultrasonographic examination. In: Naruke $T$, ed. Principle of lung cancer surgery. Vol. 3. Tokyo: Society for Study on Principle of Lung Cancer Surgery, 1991:201-29.

10. Faletra F, Ravini M, Moreo A, et al. Transesophageal echocardiography in the evaluation of mediastinal masses. J Am Soc Echocardiogr 1992;5:178-86.

11. Jakob H, Lorenz J, Clement T, et al. Mediastinal lymph node staging with transesophageal echography in cancer of the lung. Eur J Cardiothorac Surg 1990;4:355-8.

12. Machi J, Hayashida R, Kurohiji T, et al. Operative ultrasonography for lung cancer surgery. J THORAC CARDIOVASC SuRg 1989;98:540-5.

13. Musset D, Grenier P, Carette MF, et al. Primary lung cancer staging: prospective comparative study of $\mathrm{MR}$ imaging with CT. Radiology 1986;160:607-11.

14. Webb WR, Gatsonis C, Zerhouni EA, et al. CT and MR imaging in staging non-small cell bronchogenic carcinoma: report of the radiologic diagnostic oncology group. Radiology 1991;178:705-13.

15. Martini N, Heelan R, Westcott J, et al. Comparative merits of conventional, computed tomographic, and magnetic resonance imaging in assessing mediastinal involvement in surgically confirmed lung carcinoma. J Thorac Cardiovasc SuRg 1985;90: 639-48.

16. Suzuki N, Saitoh T, Kitamura S. Tumor invasion of the chest wall in lung cancer: diagnosis with US. Radiology 1993;187:39-42.

17. Glazer HS, Duncan-Meyer J, Aronberg DJ, Moran JF, Levitt RG, Sagel SS. Pleural and chest wall invasion in bronchogenic carcinoma: CT evaluation. Radiology 1985;157:191-4.

18. Pennes DR, Glazer GM, Beute GH, Lewis JJW, Madrazo BL. Limitation of CT in evaluation of neoplasms involving chest wall. J Comput Assist Tomogr 1987;11:290-3.

\section{Bound volumes available to subscribers}

Bound volumes of The Journal of Thoracic AND Cardiovascular Surgery are available to subscribers (only) for the 1995 issues from the Publisher, at a cost of $\$ 91.00$ for domestic, $\$ 118.77$ for Canadian, and $\$ 111.00$ for international subscribers for Vol. 109 (January-June) and Vol. 110 (July-December). Shipping charges are included. Each bound volume contains a subject and author index and all advertising is removed. Copies are shipped within 60 days after publication of the last issue of the volume. The binding is durable buckram with the Journal name, volume number, and year stamped in gold on the spine. Payment must accompany all orders. Contact Mosby-Year Book, Inc., Subscription Services, 11830 Westline Industrial Drive, St. Louis, Missouri 63146-3318, USA; phone 1 (800) 453-4351 or (314) 453-4351.

Subscriptions must be in force to qualify. Bound volumes are not available in place of a regular JouRval subscription. 